\title{
Lighting molded optics: Design and manufacturing
}

\author{
H. Kočárková ${ }^{1, a}$ \\ EcoGlass, a.s., Arbesova 66a, Jablonec nad Nisou, Czech Republic
}

\begin{abstract}
Proper design and manufacturing of glass molded lenses need to be performed in several steps. The whole process from customer requirements to f nal functional product is shown on two examples - a lens for street light and a lens for spot light with narrow lighting angle.

After discussing customer requirements, optical design is made. Thanks to various commercial softwares with optimization, manufacturer of the lens can work as well as a designer which enables simplif cation and acceleration of lens manufacturing, since limitations of the manufacturing process are considered during creation of the design.

When the prototype is made, its functionality needs to be evaluated. This work shows measurement of light distribution for street light lens in a dark room using goniometer and measurement of light intensity for spot lens f xed on an optical bench. These measurements can reveal the root cause in case of lens malfunction, which enables to optimize manufacturing process or modify lens design accordingly. Designing, manufacturing and evaluation of molded optics under one roof enables creation of easily manufacturable design and fast solution of problems.
\end{abstract}

\section{Introduction}

Glass lenses are nowadays used for lighting and medical applications as well as in solar business or imaging systems. This article is focused to lighting applications. Lately, the growing LED technology supports using of glass before plastic. Several companies in street lighting business offer lamps with one high power LED and properly designed glass lens. This is a new branch of lamps compared to older types, where many low power LEDs were used in a combination with plastic lenses. There are several reasons why future for glass material is brighter than for plastic in this f eld. LED is an emitter of UV light which causes degradation of plastic material. Moreover, for high power light sources, plastic cannot resist the high temperature. Another limitation for plastic lenses is their size and mass, which cannot exceed certain limit. As it will be shown later in the article, size of optics and size of light source are closely linked.

This article shows whole process of optical design from customer requirements to an existing product on two examples: lens for street light and lens for spot light. When designing lens for street lighting, light needs to be distributed into very large area. Completely different behavior of light is required in mine lamps or pocket lamps. In these cases, the lighting angle needs to be very small. Variation of lighting angles can be achieved by proper glass optics. At the beginning of the new optics development, customer requirements need to be discussed, see sec. 1 for more information. Section 2 summarizes designing of a 3D lens model and results of simulation. Production of prototypes and their experimental evaluation are discussed in sec. 3 and 4, respectively. Last section points out the most important conclusions, see sec. 5 for more details.

\footnotetext{
a e-mail: helena@ecoglass.cz
}

\section{Customer requirements}

Before we can start creation of the optical design, we need to know several key parameters such as number of the light sources and its type, distance of the light source from the optics, minimal and maximal size of optics and its material as well as size of illuminated area and its distance from the light source. First example is given for lens for street light. Key parameters are given in red in sketches below, see Fig. 1 and 2. Apart form the dimensions, it is also necessary to have data about the required illumination intensity on the target in lux units. Compared to street light, less pa-

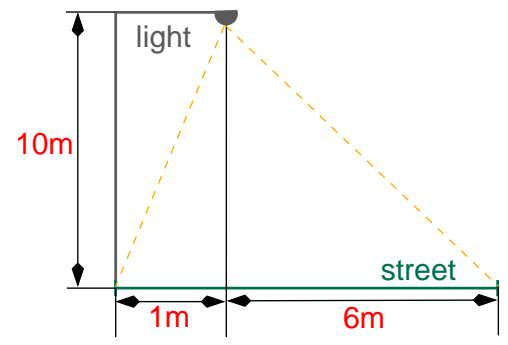

Fig. 1. Lens for street light - side view.

rameters are needed for second example with lens for spot light. Illuminated area is symmetric and lighting angle is required as small as possible. The key parameters in this task are information about light source and maximal size of lens. Lens size should not exceed certain weight since it should be used in pocket and mine lamps. Sketch is given in Fig. 3.

This is an Open Access article distributed under the terms of the Creative Commons Attribution License 2.0, which permits unrestricted use, distribution, and reproduction in any medium, provided the original work is properly cited. 


\section{EPJ Web of Conferences}

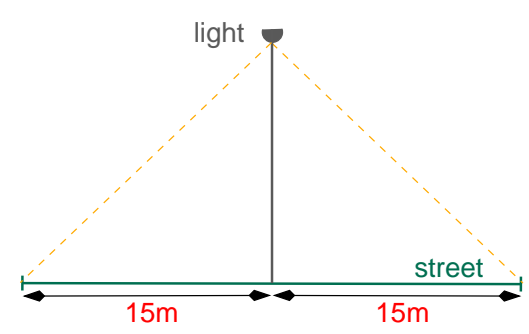

Fig. 2. Lens for street light - front view.

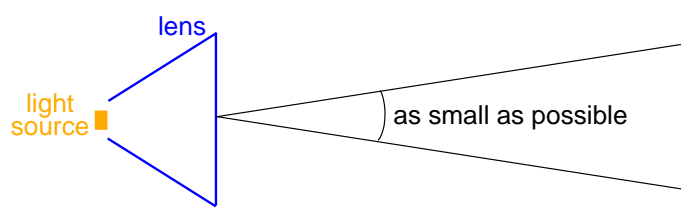

Fig. 3. Lens for spot light.

\section{Lens design}

Commercially available softwares enable designing optical components even for people who do not have deep background in optics. These softwares enable optimization of initial shape, which is proposed by designer. This brings big advantage to lens manufacturers who can design simpler lenses while considering all limitations of their manufacturing process. Moreover, when necessary to modify customer design in order to correspond with requirements for the manufacturing, the software helps to verify the optical function of lenses after necessary modif cations.

Commercial softwares were used to design lens for street light respecting the parameters mentioned in previous section and Fig. 1 and 2. Results of simulation of illuminated street with surrounded area is given in Fig. 4.

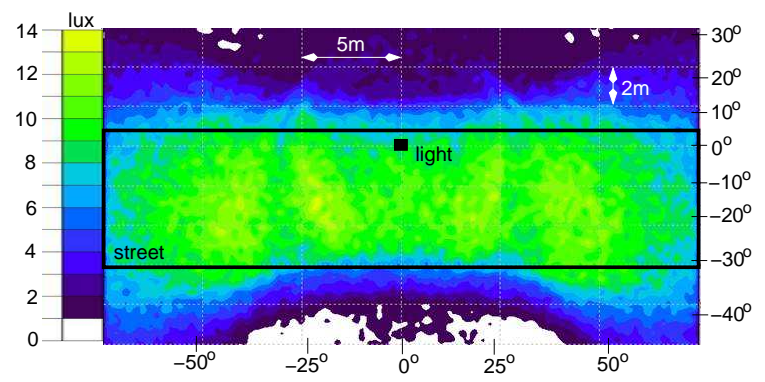

Fig. 4. Lens for street light - required illuminated area is given in black rectangular.

Square shaped surface source with size $15 \times 15 \mathrm{~mm}$ and f ux 7,000 lumen was used in the simulation. Target is in a distance $10 \mathrm{~m}$ as required. Maximal intensity is around $14 \mathrm{~lx}$. Fig. 5 shows top and bottom view of the lens model. Apart from the lighting requirements, the lens design follows several other important parameters. Lens border is big enough to enable easy mounting and thick enough to correspond with manufacturing requirements. As well the total lens size and angles strictly follow limitations of the manufacturing process. These limitations are considered while designing the initial shape and serve as limit parameters during optimization. For the frst design of spot lens, we used light source Cree XP-G, because our company already has a wide range of lenses for this light source. Spot

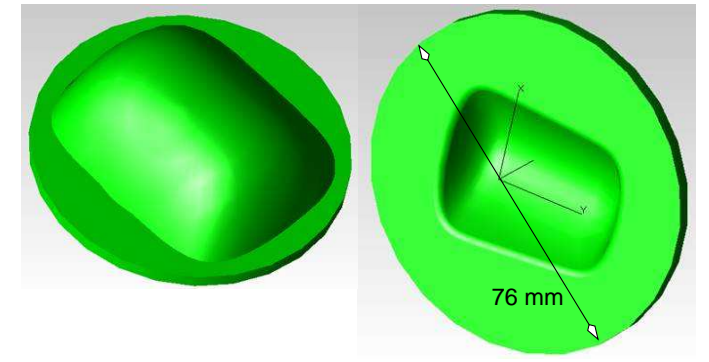

Fig. 5. Lens for street light - top (left) and bottom (right) view of the lens model in simulation program.

lenses are supposed to be used in pocket lamps and their size should remain small. Nevertheless, our f rst results showed that bigger lens gives narrower angle than smaller lens for the same light source. First lens we designed had diameter $56 \mathrm{~mm}$, second lens had diameter $70 \mathrm{~mm}$. Fig. 6 shows model of lens for spot light. Both lens sizes have the same design, only their size varies.

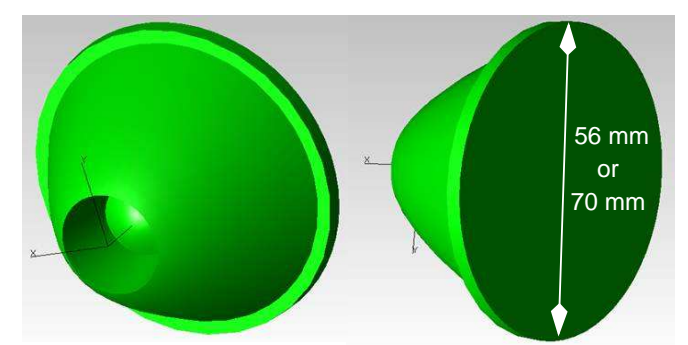

Fig. 6. Lens for spot light - back (left) and front (right) view.

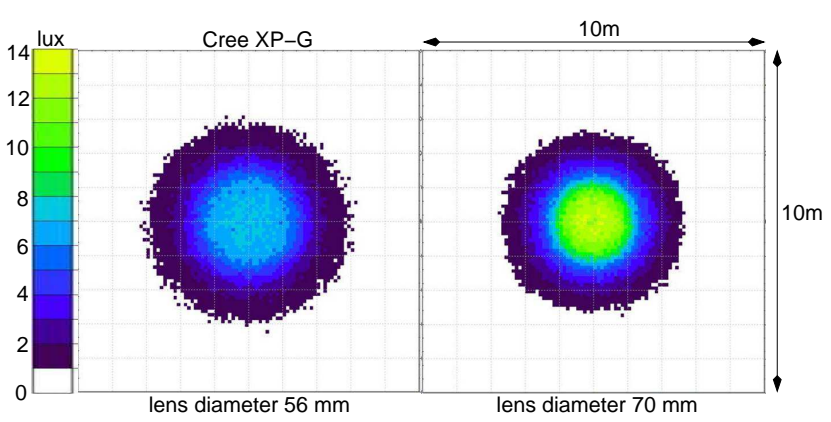

Fig. 7. Lens for spot light - comparison of two lens sizes with light source Cree XP-G. Target at a distance $50 \mathrm{~m}$ from the light source.

Fig. 7 compares illuminated area for lenses with 56 and $70 \mathrm{~mm}$ diameter and light source Cree XP-G, when target is at a distance $50 \mathrm{~m}$ from the light source. It is obvious, that lens with bigger diameter illuminates in a narrower angle. For lens with diameter $56 \mathrm{~mm}$, the angle equals to $4^{\circ}$ and for lens with diameter $70 \mathrm{~mm}$, it equals to $3^{\circ}$. The angle is taken at $50 \%$ of the maximal intensity. Maximal intensity is higher for the bigger lens and equals to 14 lx. For smaller lens is the maximal intensity $81 \mathrm{x}$. The efficiency is higher by $1 \%$ for bigger lens and equals to $96 \%$. Power of Cree XP-G was taken from data sheet and equals to $130 \mathrm{~lm}$. Because the light intensity is not very high, more powerful 
light sources were tested in combination with the two spot lenses. Fig. 8 shows results for both lenses and light source Cree XM-L with power $260 \mathrm{~lm}$. Even though the power of $\mathrm{XM}-\mathrm{L}$ is higher compared to XP-G, the maximal intensity on the target is lower than for XP-G. This can be caused by bigger size of LED chip for XM-L. Larger chip behaves less as a point source and can lead to wider lighting angle and lower maximal intensity. Therefore using XM-L results to larger lighting angle, which is now $6^{\circ}$ and $5^{\circ}$ for lenses with diameter 56 and $70 \mathrm{~mm}$, respectively. This theory is supported by lower efficiency of lenses in a combination with XM-L compared to XP-G, see Fig. 1 in Appendix A for more details.

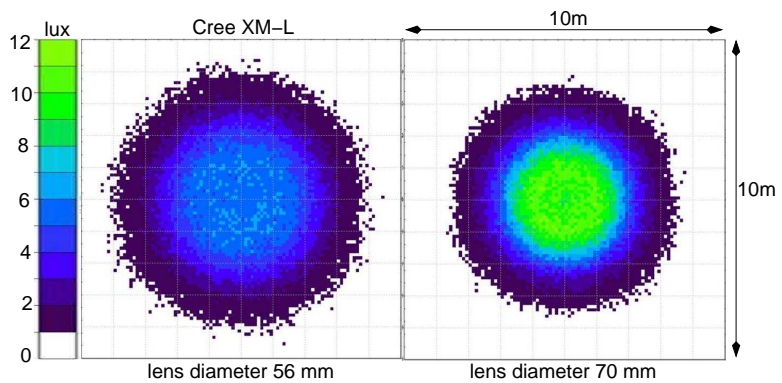

Fig. 8. Lens for spot light - comparison of two lens sizes with light source Cree XM-L. Target at a distance $50 \mathrm{~m}$ from the light source.

Fig. 9 shows results of simulation for both lenses in a combination with even more powerful LED, which is Luminus SST-90 with power $1200 \mathrm{~lm}$. Bigger chip size causes larger lighting angle, but since the LED power is much higher compared to both Cree LEDs, the maximal intensity equals to $28 \mathrm{~lx}$ for bigger lens and is twice as high as for Cree XP$\mathrm{G}$. In comparison, light efficiency is much smaller which is again result of large LED chip. Comparing results in Fig.

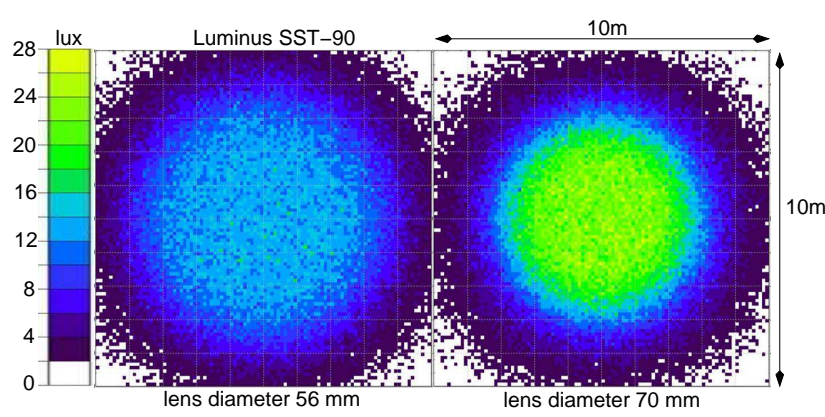

Fig. 9. Lens for spot light - comparison of two lens sizes with light source Luminus SST-90. Target at a distance $50 \mathrm{~m}$ from the light source.

7,8 and 9 shows that growing size of light source causes bigger lighting angle, because the light source behaves less and less as point source. The maximal intensity grows with increasing power of the light source, but only if the LED power is much higher, otherwise size of the chip is a dominant parameter, which influence the maximal intensity. Summary for spot lenses and all light sources including information about lighting angle, light efficiency and maximal intensity is given in Fig 1 in Appendix A.

\section{Production of prototypes}

Our products are molded from pre-heated glass rods. Lens for street light is manufactured using both-sides molding process. The top part of these products as well as the bottom part are only molded and the rest of the glass is removed by grinding. Distance between the two mold parts needs to be sufficient to ensure proper molding. This is a key parameter for lens border.

Spot lens is an example of product with all optically functional surfaces on one side and fla polished surface on the other side. Polished surface has better quality than molded surface and supports good optical function, but it can increase the lens price.

It is necessary to control the molding process and pay special attention to optically functional surfaces during the production. This is easier when the manufacturer is also the lens designer. Fig. 10 shows in black the most important surface of spot lens that collimates light in narrow angle. Special attention is paid to molding of this surface since every deformation influence highly the optical function, as it will be shown in the following section.

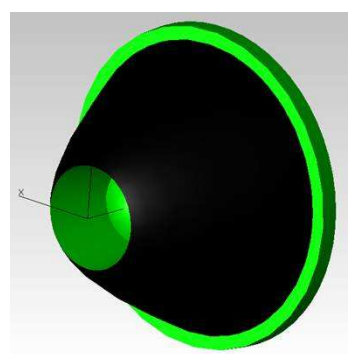

Fig. 10. Lens for spot light - black surface reflect most of light and creates narrow lighting angle.

Prototypes of lens for street light as well as prototypes of spot lenses with diameter 56 and $70 \mathrm{~mm}$ are given in photos in Fig. 11.

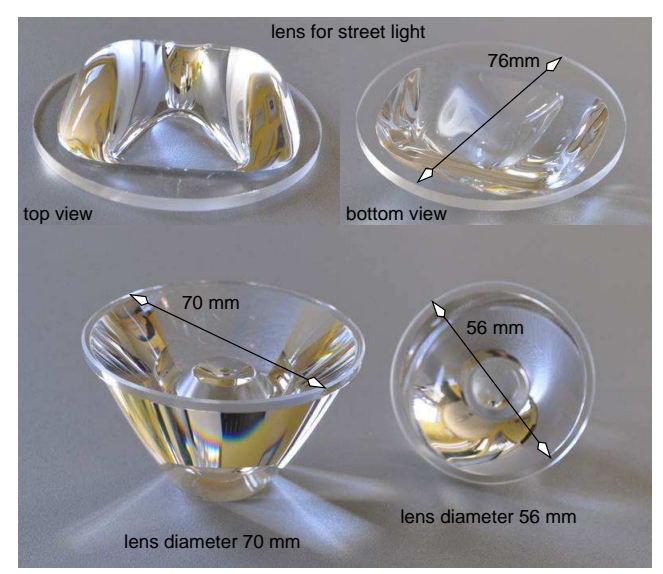

Fig. 11. Prototypes of lens for street light and both lenses for spot light. 


\section{Experimental evaluation}

Evaluation of lens function is in our company carried out in two main ways. One is measurement of light intensity when lens and light source are fxed on an optical bench. The second option is measurement of illumination map using goniometer in dark room. This method was used for determination of illumination map of lens for street light, see Fig. 12. Results were obtained in a dark room, where test-

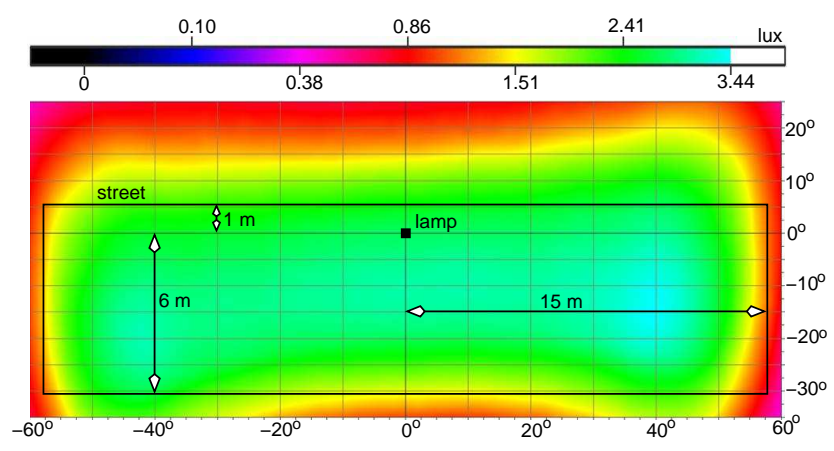

Fig. 12. Illumination map of lens for street light measured in a dark room via goniometer and luxmeter.

ing distance is $10 \mathrm{~m}$. Comparing light distribution in Fig. 4 and Fig. 12 shows good agreement of simulation result and real measurement. The maximal intensity is much lower for experimental measurement, because the light source in simulation was expected to have 7,000 lumen, while in reality LED with approximately 3,000 lumen was used. When the maximal intensity for 7,000 lumen is $14 \mathrm{~lx}$ in simulation, it should be approximately $61 \mathrm{x}$ for 3,000 lumen in the experimental determination. The maximal measured intensity was $3.5 \mathrm{~lx}$, which is $60 \%$ of the theoretical value, but since we cannot be certain about the real f ux 3,000 lumen, the predicted efficiency of lens compared to simulation is mainly informative.

Lenses for spot light were evaluated on an optical bench, see Fig, 13. Light source is f xed on a support, which vertical and horizontal position can be changed via several screws. Other screws also enable small tilt of the light source. Lens is placed in a plastic case and centered in a holder, which is $\mathrm{f} x e d$ to a micro rotation stage. This enables changing lens position in a direction to the light source as well as rotating the lens. Lens and source can be set to the correct mutual position thanks to this equipment. Maximal intensity was measured on a target at distance $5 \mathrm{~m}$ from the light source. Tab. 1 summarizes experimentally determined maximal light intensities for lenses in a combination with various light sources. Compared to simulation, the highest intensity is achieved for Cree XP-G. Standard amperage

Table 1: Measured maximal intensities in lux for lenses for spot light and various light sources.

\begin{tabular}{|c|c|c|c|}
\hline & $\begin{array}{c}\text { Cree } \\
\text { XP-G }\end{array}$ & $\begin{array}{c}\text { Cree } \\
\text { XM-L }\end{array}$ & $\begin{array}{c}\text { Luminus } \\
\text { SST-90 }\end{array}$ \\
\hline $\begin{array}{c}\text { spot lens with } \\
\text { diameter 70 mm }\end{array}$ & 1147 & 737 & 563 \\
\hline $\begin{array}{c}\text { spot lens with } \\
\text { diameter 56 mm }\end{array}$ & 755 & 450 & 333 \\
\hline
\end{tabular}

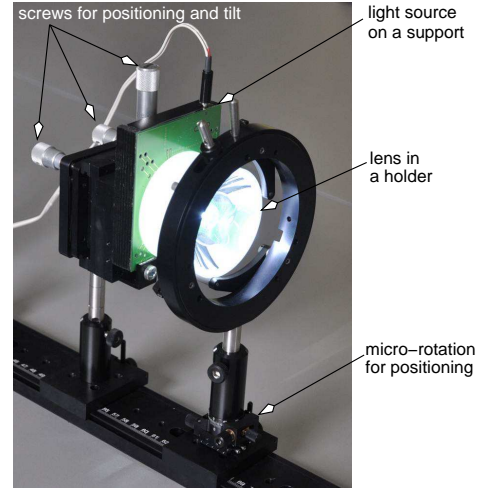

Fig. 13. Experimental set-up on an optical bench for determination of maximal intensity of lenses for spot light.

of Cree XP-G is $350 \mathrm{~mA}$ which corresponds to total $\mathrm{fux}$ $130 \mathrm{~lm}$ and was considered in the simulation. During experimental evaluation, module with 1.2 A was used. Higher amperage leads to higher total $\mathrm{f} u \mathrm{x}$ of the light source and according to the data sheet, it shall correspond to $364 \mathrm{~lm}$. Same amperage was used in a module with Cree XM-L, which leads to an increase of the total $\mathrm{f} \mathrm{ux}$ from $260 \mathrm{~lm}$ at amperage $700 \mathrm{~mA}$ to approximately $416 \mathrm{~lm}$. Standard amperage for Luminus SST-90 is $3.15 \mathrm{~A}$, but in our experimental evaluation, module with lower amperage of $1.8 \mathrm{~A}$ was used. The decrease of amperage causes decrease in the total $\mathrm{f} \mathrm{ux}$ from $1200 \mathrm{~lm}$ to only $686 \mathrm{~lm}$. The total $\mathrm{f} \mathrm{ux}$ for Cree XP-G, XM-L and Luminus SST-90 therefore equaled to 364,416 and $686 \mathrm{~lm}$, respectively during the experimental evaluation. Since all values are of the same grade, size of the LED chip is a dominant parameter that inf uences the maximal intensity and explains why the highest intensity was experimentally determined for XP-G and lowest intensity for SST-90. Inf uence of the chip size was discussed earlier in sec. 2 .

We can compute theoretical value of maximal intensity that shall appear on a target during experimental evaluation from simulation results given in sec. 2. Considering lower total f ux and shorter distance for experimental measurement, we obtain maximal intensities for XP-G, XM-L and SST-90 as follows: 3,920 lx, 1,920 lx and 1,600 lx. Maximal measured intensity for XP-G was $1,147 \mathrm{~lx}$, which is approximately $30 \%$ of the theoretical value. Similar results were obtained for the two other light sources. For XM-L is the maximal measured intensity only $38 \%$ of the theoretical value and for SST-90 it is roughly $35 \%$. This big loss in intensity can be explained by Fresnel ref ection, incorrect mutual position of lens and light source, imperfections on the optical surface created during molding process or error of measurement. To conf rm the new computed f ux of LED modules used in the experimental evaluation, intensity of light source without the optics was measured and compared to result obtained the same way in simulation. For XP-G, the theoretical maximal intensity on the target shall be $8.4 \mathrm{~lx}$, but equaled only to $5.5 \mathrm{~lx}$ during the experimental measurement. The loss of total $\mathrm{fux}$ without the optics is therefore $35 \%$ and suggest that the recomputed total f ux may not correspond with reality. Value of the maximal intensity should be considered as informative and not an exact number. 
Unlike the determined max. intensity, lighting angle at $50 \%$ of maximal intensity corresponds nicely with the simulation. Angles can be computed using values in Tab. 2, where the measured illuminated area and theoretical illuminated area are compared.

Table 2: Diameter of measured and theoretical light circle for both spot lenses and all tested light sources.

\begin{tabular}{|c|c|c|c|}
\hline $\begin{array}{c}\text { light } \\
\text { source }\end{array}$ & $\begin{array}{c}\text { lens } \\
\text { diameter }\end{array}$ & $\begin{array}{c}\text { measured } \\
\text { circle }\end{array}$ & $\begin{array}{c}\text { theoretical } \\
\text { circle }\end{array}$ \\
\hline Cree & $56 \mathrm{~mm}$ & $35 \mathrm{~cm}$ & $35 \mathrm{~cm}$ \\
XP-G & $70 \mathrm{~mm}$ & $23 \mathrm{~cm}$ & $28 \mathrm{~cm}$ \\
\hline Cree & $56 \mathrm{~mm}$ & $51 \mathrm{~cm}$ & $52 \mathrm{~cm}$ \\
XM-L & $70 \mathrm{~mm}$ & $41 \mathrm{~cm}$ & $42 \mathrm{~cm}$ \\
\hline Luminus & $56 \mathrm{~mm}$ & $80 \mathrm{~cm}$ & $79 \mathrm{~cm}$ \\
SST-90 & $70 \mathrm{~mm}$ & $62 \mathrm{~cm}$ & $61 \mathrm{~cm}$ \\
\hline
\end{tabular}

It was found out that LED chip is projected around the maximal intensity measured in the middle of the round shape. Square shaped illuminated area is visible in Fig. 14 on the left. When a small round object is placed in the middle of the lens, chip projection is eliminated, see right image in Fig. 14. Nevertheless, the elimination of the LED chip projection causes decrease of intensity by approximately $8 \%$.

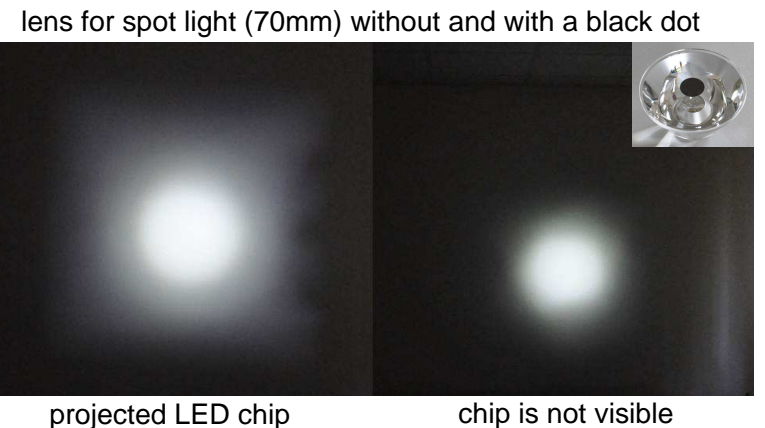

Fig. 14. $70 \mathrm{~mm}$ lens for spot light - illuminated area with a projected LED chip (left) and without (right), where rays from the middle part of the lens were shaded.

As it was mentioned in sec. 3 and Fig. 10, it is important to pay attention to optically functional surfaces during molding. Experimental evaluation on an optical bench can reveal defects on the molded surface. Fig. 15 compares a $70 \mathrm{~mm}$ lens with defects on the molded surface (left) with a properly molded lens (right). Illuminated area in the right image has perfectly round shape of high intensity and square shaped illuminated area of lower intensity, which is caused by the projected chip. In comparison, the illuminated area in the left image is not perfectly round, but blurry. This is result of deformation on the optically functional side.

\section{Conclusion}

Process from customer requirements to experimental evaluation of prototype was described in details in this article. Design of lens for street light was modifie in commercial

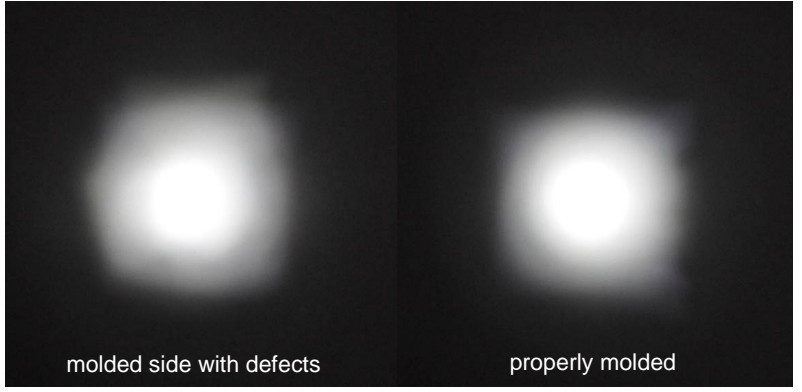

Fig. 15. $70 \mathrm{~mm}$ lens for spot light - illuminated area with lens with defects on the molded surface (left) and properly molded lens (right).

program until it fulfille all requirements. Since all production limitations were considered during the designing process, no problems occurred during manufacturing and also experimental evaluation of the firs prototypes in dark room showed good agreement between the simulation and real measurement. This proves how big advantage is when the designer is also manufacturer and how efficiently is the time spent between the initial idea and real product.

Second example with designing lens for spot light showed close link between the lens size and LED chip. With a growing size of LED chip, maximal intensity and efficiency are decreasing. In comparison, the lighting angle is increasing. Maximal intensity can rise only if the power of larger LED chip is much bigger than the power of smaller chip. It will be very interesting to investigate these relations in details in the future.

Bigger lens for spot light is on the limit of manufacturing. Higher attention has to be therefore paid to optically functional surfaces during molding.

Maximal intensity was lower in the real measurement compared to simulation, which is most probably caused by low amperage of the light sources used during experimental evaluation. The lower maximal intensity also appears as a result of Fresnel reflection incorrect mutual position of lens and light source and imperfections on the optical surface. In comparison, size of the illuminated area nicely corresponds with results from simulation. While the maximal intensity can be easily modifie by change of amperage, lighting angle is a property of the lens and therefore it is more important that especially this parameter corresponds with results from simulation.

This article shows how having designing, manufacturing and evaluation under one roof enables to work fast and remain focused to important parts of each step in creation of molded optics for lighting.

More information at: www.ecoglass-optic.com 


\section{Appendix A}

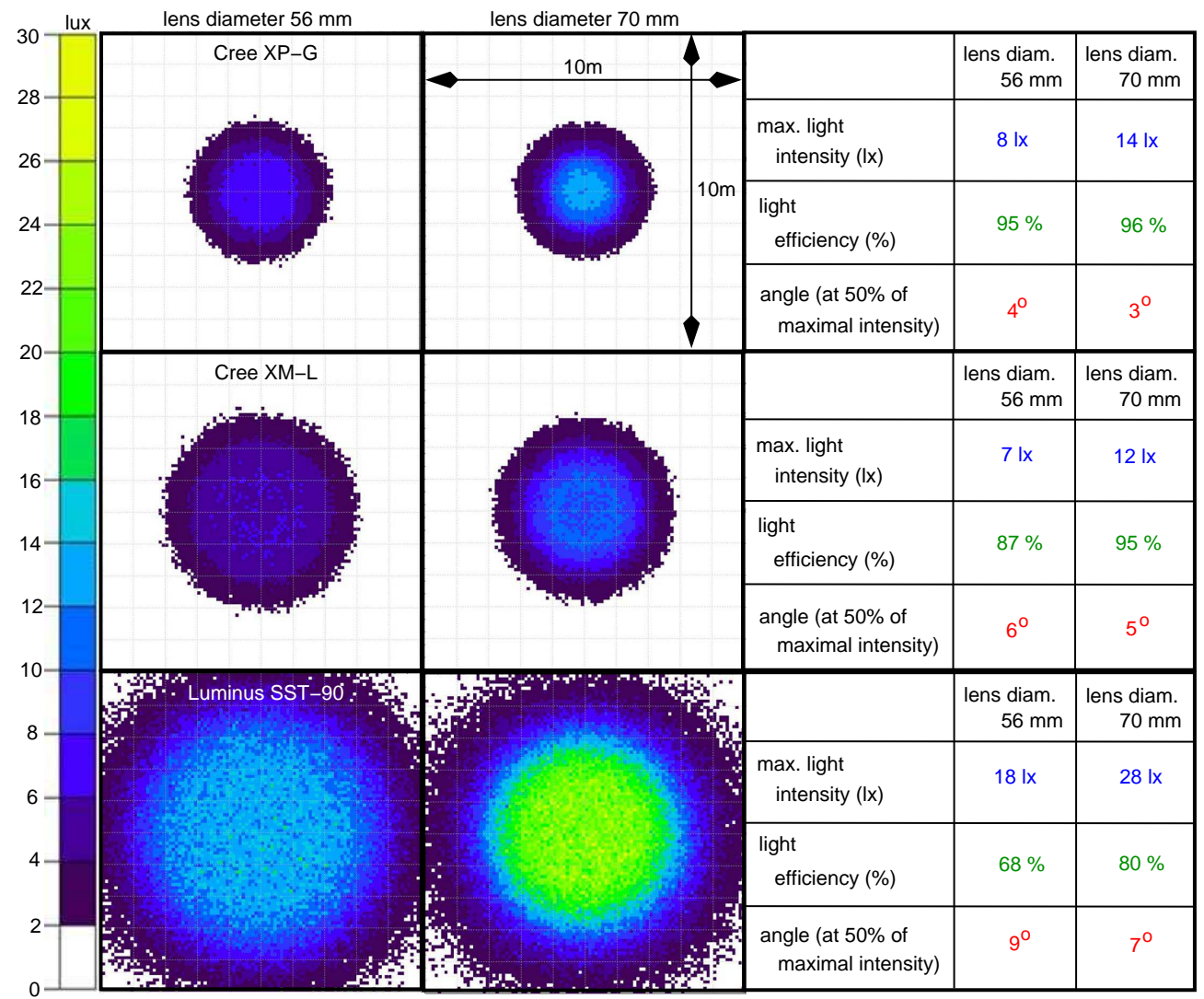

Fig. 1. Summary of results for spot lenses with diameters 56 and $70 \mathrm{~mm}$ in a combination with various light sources. 